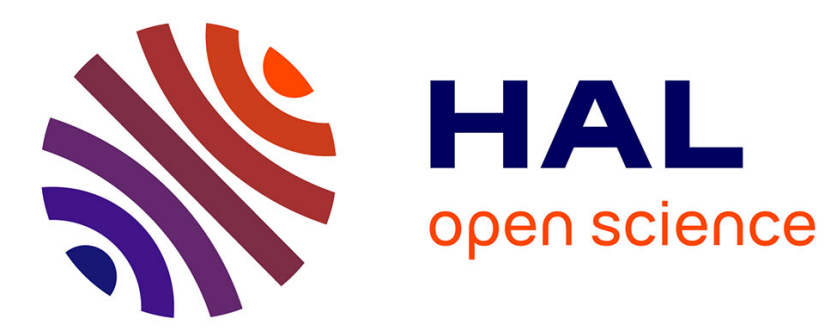

\title{
Inscription sur bronze de Thoard (Basses-Alpes)
}

Henri Rolland

\section{To cite this version:}

Henri Rolland. Inscription sur bronze de Thoard (Basses-Alpes). Gallia - Fouilles et monuments archéologiques en France métropolitaine, 1960, 18 (1), pp.103-109. 10.3406/galia.1960.2291 . hal01925954

\section{HAL Id: hal-01925954 \\ https://hal.science/hal-01925954}

Submitted on 25 Feb 2020

HAL is a multi-disciplinary open access archive for the deposit and dissemination of scientific research documents, whether they are published or not. The documents may come from teaching and research institutions in France or abroad, or from public or private research centers.
L'archive ouverte pluridisciplinaire HAL, est destinée au dépôt et à la diffusion de documents scientifiques de niveau recherche, publiés ou non, émanant des établissements d'enseignement et de recherche français ou étrangers, des laboratoires publics ou privés.

\section{(이) $\$$}

Distributed under a Creative Commons Attribution - NonCommercial - NoDerivatives| 4.0 


\title{
INSGRIPTION SUR BRONZE DE THOARD
}

\author{
(Basses-Alpes)
}

\author{
par M. Henri Rolland
}

Dans le tome XII du Corpus inscriptionum latinarum, O. IIirschfeld signalait l'absence totale d'inscriptions provenant de Digne (Basses-Alpes)1 ${ }^{1}$; cette carence n'est plus aujourd'hui absolue, à la suite d'une découverte ${ }^{2}$ faite fortuitement en octobre 1956. A cette époque, M. Guy Delaye procédait à des labours, sur un coteau exposé au Sud, dans un champ dépendant de la ferme dite Plan de Silve située à Thoard, quartier de Franco, à $2 \mathrm{~km} .500 \mathrm{du}$ village, dans la direction de Saint-Estève, et à environ 10 kilomètres de Digne. A une profondeur de $0 \mathrm{~m}$. 50, il rencontra quelques ossements et deux fragments d'une plaque de bronze dont l'un portait une inscription malheurcusement très mutilée (fig. 1). A ces vestiges était associé un objet en plomb, sorte de spatule dont la partie convexe est ornéc d'incisions concentriques et disposées en chevrons. L'inventeur ayant prévenu de sa trouvaille M. Raymond Collier, archiviste en chef des Basses-Alpes, celui-ci, après avoir obtenu le don gracieux de l'inscription pour le musée de Dignc, procéda, avec la collaboration de M. R. Moulin, à des fouilles complémentaires sur le lieu de la découverte ; là furent recueillis quelques objets et débris déplacés ou brisés par le passage de la charrue :

une pointe de fer brisée, à tête de bronze en forme de pommeau; deux pointes de javelot en fer, l'une intacte de $0 \mathrm{~m}$. 243 à pointe triangulaire $(0 \mathrm{~m}$. 145) munie d'une douille conique ; l'autre brisée (0m. 23), formée d'une pointe quadrangulaire sur douille conique ; - un coutelas en fer, à lame légèrement courbe, le manche terminé par un crochet très ouvert, le lout d'une seule pièce, mesurant dans son état actuel $0 \mathrm{~m}$. 245 ; - un anneau de fer, de $0 \mathrm{~m}$. 022 de diamètre, appartenant peut-être à l'une des pièces précédentes; - - une sonnaille, constituće par deux pièces de fer assemblées par des rivets, privée de sa bélière brisée, haute de $0 \mathrm{~m}$. $065 ;$ - des clous de fer ; - une petite "boîte à fard" en forme de lampe, en bronze, de $0 \mathrm{~m} .039$ sur $0 \mathrm{~m} .022$; - des tessons de vases ; poteries de cuisine à pâte grise ou noirâtre dure, à fonds plats, parois renflées, lèvres retournées vers l'extérieur, provenant d'une dizaine de vases ; poterie ordinaire en argile jaunâtre ou rougeâtre ; une coupe en terre fine, rouge, non sigillée, haute de $0 \mathrm{~m}$. 10 et de $0 \mathrm{~m}$. 30 de diamètre, portant à l'extérieur du fond le graffite $M$; les débris d'un dolium ; des fragments d'un récipient grossier, d'une amphore et de tuiles; - deux sesterces de bronze, l'un, en mauvais élat au nom de Faustine mère, l'autre de Gordien III, à la légende IMP GORDIANVS PIVS FEL AVG. -- AETERNITATI AVG SC, recouvert d'une belle patine bleu-vert et en très bel état.

(1) Le Musée de Digne conserve une courte inscription romaine insignifiante, dont la découverte, également à Thoard, demeure très incertaine.

(2) Signalée dans les Informations de "Gallia", t. XVI, 1958, p. 400. 


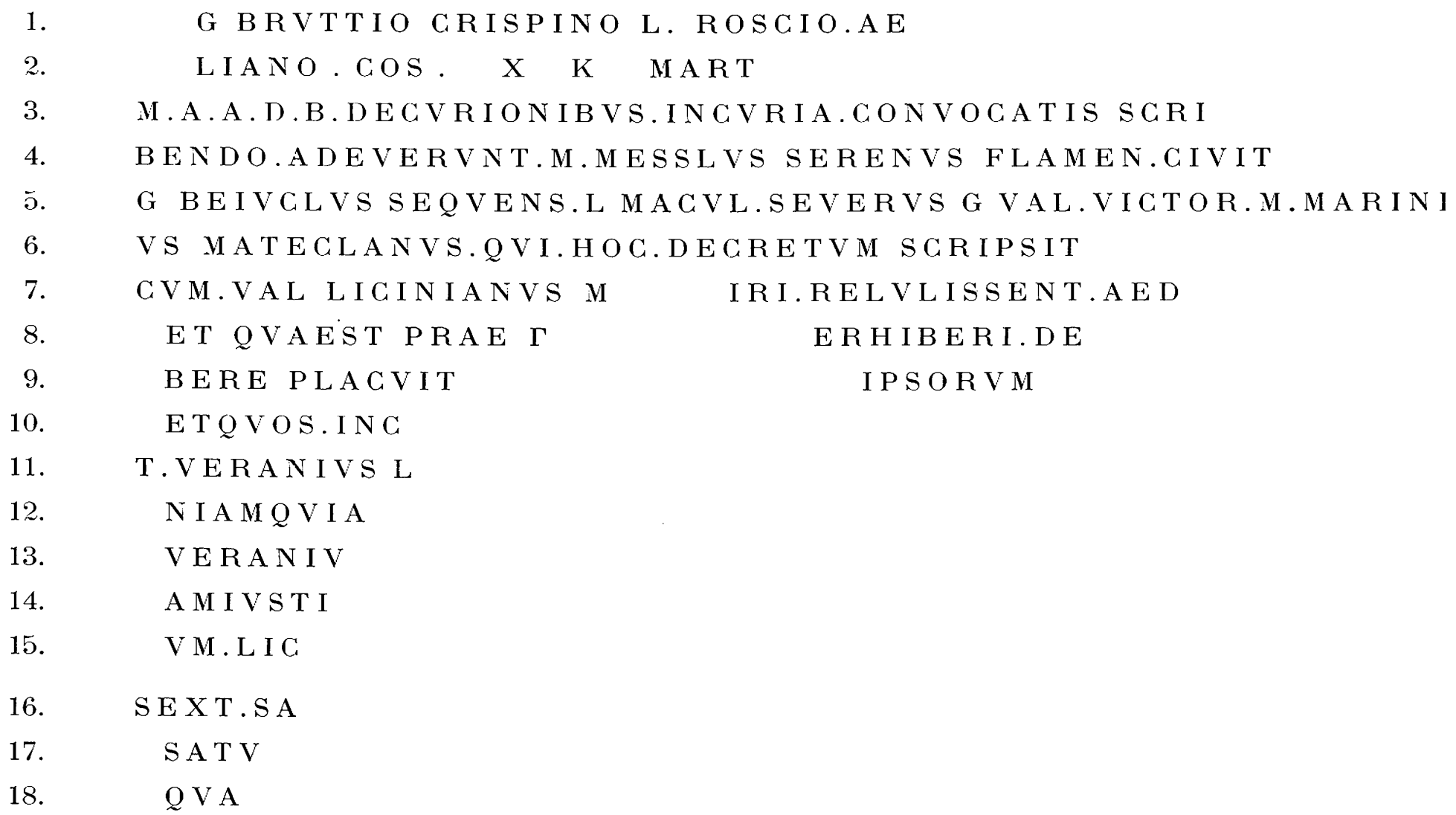


C,BRUTHIOCRISPINOLROSCIOXE $11 \lambda=10.20 S \times K \lambda \lambda R$

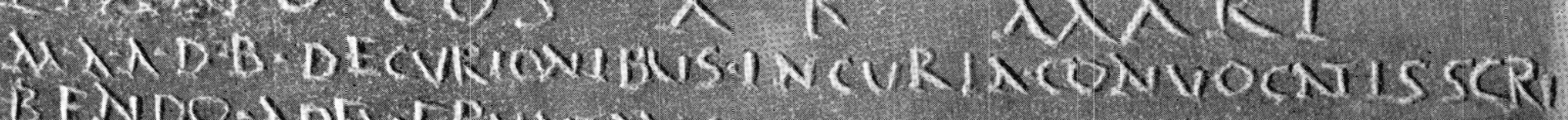

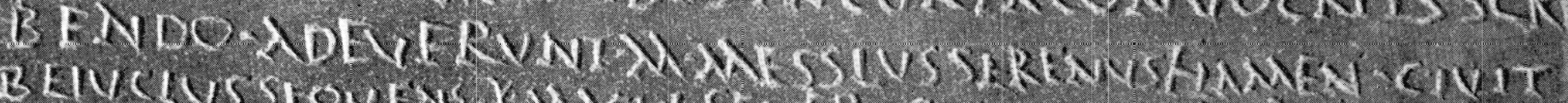

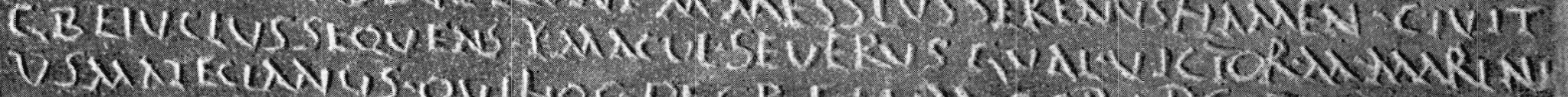

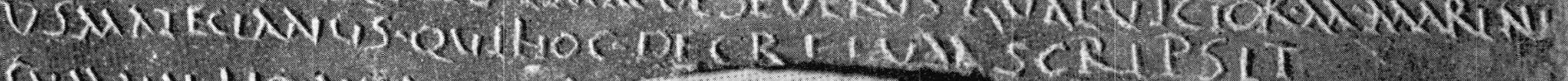

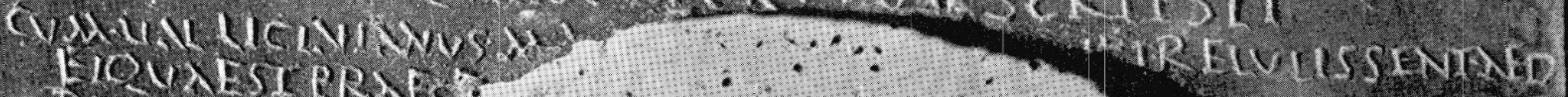

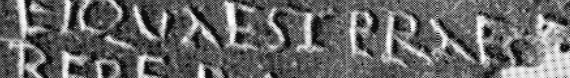

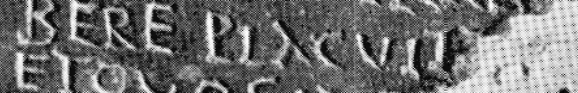

1. VERTIIVS Nintevis VIR 1 visy? Xuvisi: villes

S Exis is $5 \times 1 \times$ $\Omega \wedge$

Fig. 1. - Décret des décurions de Digne, 187. Plaque de bronze trouvée à Thoard (Basses-Alpes). (Clichó Audouard). 
Cet inventaire montre qu'il ne s'agit pas d'une sépulture, comme nous l'avions pensé au début. Cependant l'absence totale de substructions de maçonnerie, sur une surface étendue, éloigne l'hypothèse d'une construction ayant quelque importance; on en est réduit à voir là une simple cabane, en grande partie en bois, comme l'indique la présence de nombreux clous (70 environ) trouvés dans une couche charbonneuse; sans doute, traces d'un incendic postéricur au III $^{\mathrm{e}}$ siècle si l'on tient compte du terminus post quem que constitue la monnaie de Gordien III (238-244). Cet abri rustique cadre mal avec la présence d'une inscription sur bronze ; il faut admettre que cette plaque y a été transportée d'ailleurs à une fin utilitaire, accusée par la mutilation qui n'est pas le fait d'une brisure accidentelle mais d'un découpage à l'aide d'un outil.

Cette plaque, épaisse de $0 \mathrm{~m}$. 003, large de $0 \mathrm{~m}$. 21, est incomplète dans sa hauteur actuelle $(0 \mathrm{~m} .146)$, comme en témoigne l'interruption inférieure de la bordure encadrant le texte, dont une partie essentielle a disparu (fig. 1). Cependant, malgré son état fragmentaire, ce document épigraphique mérite, à plus d'un titre, de retenir l'attention. C'est la première inscription trouvée sur l'antique territoire de la cité de Digne et, de plus, la première qui la conccrne; c'est aussi l'une des rares inscriptions attestant, en HauteProvence, une organisation municipale analogue à celle des villes gallo-romaines de la Narbonnaise méridionale et occidentale.

Le texte ne comprend que six lignes complètes ; douze autres sont mutilées et progressivement réduites jusqu'à ne plus comprendre que trois lettres. Aussi l'interprétation présente-t-elle des difficultés insurmontables, sauf à son début qui, à lui seul, mérite d'être étudiè. Il s'agit d'un document rare, un décret des décurions d'un municipe qui, bien que désigné sous ses seules initiales, ne peut s'identifier qu'avec Digne.

Le texte est entouré d'une marge formant encadrement à profil de doucine, limitée vers l'intérieur par une baguette. Il est écrit en capitales convenant au II ${ }^{\mathrm{e}}$ siècle ; on $\mathrm{y}$ remarque les A à branche gauche venant se souder bas sur la branche droite avec suppression de la barre transversale ; les B à boucle inférieure plus développée que la boucle supérieure ; les $\mathrm{G}$ en forme de $\mathrm{C}$, suivi d'une virgule ; les II ayant la barre transversale légèrement inclinéc ; les $\mathrm{L}$ à barre inférieure ayant tendance à descendre au-dessous de la ligne ; les $\mathrm{M}$ à jambages écartés à tendance cursive; les $\mathrm{R}$ à petite boucle et queue développéc ; les $\mathrm{V}$ à branche gauche légèrement incurvée. On a là un nouvel exemple ${ }^{3}$ de mauvaise transcription du modèle manuscrit par un graveur qui confond $\mathrm{I}, \mathrm{L}$ et $\mathrm{T}$, ainsi que $\mathrm{E}$ et $\mathrm{F}$.

La rédaction du document est en tout conforme à la diplomatique des décrets retrouvés en Italie et sur le domaine romain; les deux premières lignes ont été tracées en caractères plus apparents que ceux employés pour le corps du texte, elles font connaître la date consulaire :

[1. 1-2] G(aio) Brultio Crispino L(ucio) Roscio Aeliano Co(n)s(ulibus) X. K(alendas) Marl(ias).

(3) J. Marı.on, Paléographie romaine, 1952, p. 57 et s. a insisté sur les deux opérations successives qui aboulissent à l'achèvement d'un texte épigraphique : l' ordinatio" et la gravure ; c'est entre ces deux étapes que se produisent les confusions. 
Le 10 avant les calendes de mars, soit le 20 février, sous le consulat de Caius Bruttius Crispinus et de Lucius Roscius Aelianus, correspondant à l'année 187. On possède plusieurs inscriptions mentionnant ce consulat ${ }^{4}$, dont deux décrets datés de Pouzzoles du 5 des calendes de novembre et du 15 de celles de janvier ${ }^{5}$. Tous ceux de ces documents qui donnent le prénom du premier consul le nomment Lucius et non Caius comme sur le nouveau texte ; Caius est le prénom de son père ; il peut y avoir eu confusion dans une localité éloignée, mais le fait ne doit pas surprendre, car il existe d'autres exemples d'une étrangc dualité de prénoms où celui du père est joint à celui du fils ou le remplace, l'un ou l'autre étant indifféremment employé ${ }^{6}$. Lucius Bruttius (Quintius) Crispinus ${ }^{7}$ était fils de Caius Bruttius Praesens et frère de Crispine, femme de l'Empereur Commode.

Le second consul, Lucius Roscius Aelianus (Paculus) ${ }^{8}$, avait été en 170 flamen collegii Salii Palatini ${ }^{9}$, marié à Vibia Salvia Varia, nièce de Dide Julien; elle appartenait à la lignée du jurisconsulte Salvius Julianus ${ }^{10}$.

[1. 3] M[unicipio] A. A. D[iniensium] $B$ [odionticorum] Decurionibus in curia convocatis.

Après la date suit l'indication de la ville où a été rédigé le décret sur l'accord des décurions convoqués à la Curie ${ }^{11}$. On se heurte ici à une difficulté que présente la restitution des cinq initiales désignant la localité. Trois de ces lettres peuvent aisément être complétées, $M$. mis pour municipium et $D$. B. pour Diniensium Bodionlicorum. La ville de Digne, qui se trouvait sur la frontière des Alpes-Maritimes, avait primitivement fait partie de cette province ; clle en fut détachée par Galba, en 69, pour être annexée à la Narbonnaise où elle demeura jusqu'à la fin du II $^{\mathrm{e}}$ siècle, époque à laquelle elle se trouve à nouveau situéc dans les Alpes-Maritimes par la Notitia Galliarum ${ }^{12}$. Elle fut élevée au rang de cité, vraisemblablement au début de l'Empire; avec juridiction sur la vallée de la Bléone, dans les limites des frontières qui seront conservécs par le premier diocèse. Son nom Dinia est donné par Pline $^{13}$ à l'oppidum des Bodionticicit ${ }^{14}$ ceux-ci s'identifient avec les Brodontii ${ }^{15}$ cités sur l'inscription du Trophée d'Auguste à La Turbie. La forme normale Bodionticus est attestée par deux inscriptions de Dalmatie concernant des soldats de la Cohors III Alpinorum ${ }^{16}$.

(4) C. I. L. III supp., 8196 ; VI, 8775 ; VIII, 24125 ; XIII, 8185, 11757.

(5) C. I. L. X, 1784 ; VI, 8775.

(6) A. Chement pallu de lessi:rt, Le consulal du jurisconsulte Salvius Julianus el le syslème des prénoms multiples, Centenaire de la S.N.A.F. 1904, pp. 369-375. Salvius Julianus, proconsul d'Afrique en 148, porte indifféremment les prénoms de Publius et de Lucius.

(7) C. I. L. VI, 7582.

(8) C. I. L. XV, 7523.

(9) C. I. L. VI, 1978.

(10) Le fils du consul de 187, lui-même consul en 232, avait ajouté à son nom celui de Salvius Julianus; cf. RealEncyclopädie, I A 1, c. 1119, no 9.

(11) Rapprocher la formule d'un décret de Gabii: in municipio in curia Aelia Augusta..., C. I. L. XIV, 2795. Pour la convocation à la curie, voir C. I. L. IX, 3429; X, 460, 476, 477, 1453.

(12) Otto Seeck, Nolitia Galliarum, Berlin, 1876, p. 273, XVII, 3 : In provincia Alpium... civilas Diniensium.

(13) Hist. Nat., III, 5.

(14) HoLner, Altceltischer Sprachschatz, t. I, pp. 455-456. *Bodiontii>Bodiontici rapproché du gentilice Bodonius comme Voconlius de Voconius, Ilontia de Ilonia; la base *Bodi entrant dans d'autres composés.

(15) L'adjonction de l'r résultant d'un pléonasme graphique ou d'une alternance due à un phénomène de substrat, cf. N. Lamboglia, dans Revue des Études Ligures, 1944, p. 41.

(16) C. I. L. III, 8495, 9007. 
Reste à tenter une explication $\operatorname{des} \operatorname{deux} \Lambda$. $A$. qui suivent l'm de municipium; on ne peut que présenter des hypothèses. L'une d'clles pcut s'autoriser de la présence, à Digne, de vestiges antiques près d'une source thermale pour proposer de retrouver dans le premier $A$ le mot $A$ (quae) suivi d'un qualificatif commençant par $A$ comme on en a de nombreux exemples ${ }^{17}$. Cependant il parait plus probable que les deux $A$ sont en rapport immédiat avec le municipium qui, comme tant de colonies et de villes ${ }^{18}$, aurait été décoré d'un titre impérial : municipium Aurelium Antoninianum ou municipium Aelium Aurelium, ce dernier pouvant parfaitement convenir à la date du décret de 187, publié sous le règne de Commode.

[1. 3-6] Scribendo adfuerunt M(arcus) Messius Serenus flamen civit(alis) G(aius) Beiuclus Sequens L(ucius) Macul Severus G(aius) Val(erius) Victor M(arcus) Marinius qui hoc decrelum scripsit.

Précédée de la formule consacrée scribendo adfuerunt ${ }^{19}$, suit la liste des décurions siégeant à l'assemblée. En tête figure celui qui, par ses fonctions religieuses, semble désigné pour la présidence, Marcus Messius Serenus flamen civilatis ${ }^{20}$. Il appartient à une illustre famille répandue dans les Gaules ${ }^{21}$ et particulièrement en Narbonnaise ${ }^{22}$; on pense que certains de ses membres habitaient à Vaison une riche villa et l'on possède, à Arles, l'épitaphe ${ }^{23}$ d'une Julia Serena ${ }^{24}$, femme d'un Messius Sym..., qui pourrait bien être la mère du flaminc.

Gaius Beiuclus Sequens a son nom de famille certainement mal transcrit par le graveur ; bien qu'il y ait, connu ailleurs, un Bellucius ${ }^{25}$ d'origine celtique, il est vraisemblable qu'il faut rétablir la graphic Betutius que l'on rencontre près de Grenoble, à Uzès, à Nimes et à Narbonne ${ }^{26}$.

Lucius Macul Severus, si son patronyme n'a pas été abrégé et déformé par le graveur, paraît être d'origine indigène, son nom se rapprochant de la racine celtique Mac ou Macc.

Gaius Val(erius) Victor ${ }^{27}$ : l'abréviation de son patronyme pourrait être aussi bien complétée en Valerianus; cependant l'existence d'un $C$. Valerius Victor et d'un L. Valerius Victor plaide en faveur de la restitution adoptéc.

(17) Aquae Apollinares, Aquae Auguriae, Aquae Auguslae, Aquae Aureliae, etc.

(18) Colonia Julia Aurelia Anloniniana, Colonia Augusia Anloniniana, Colonia Aelia Aurelia Augusta, etc.

(19) C'est là une formule constante dans les décrets : C.I. L. IX, $3429 ; \mathrm{X}, 1784$, à Pouzzoles sous le même consulat, scribundo adfuerunl; X, 476, 477, à Paestum, in curia... decuriones adfuissent; X, 3698, à Cumes, scribundo sorle ducli adfuerunt; X, 1453, 4760, à Suessa, in curia scribendo adfuerunl; XI, 1924, in schola Laeliana scribundo adfuerunt. Le texte de Digne porte adeuerunl, cacographie due au graveur confondant sur le modèle manuscrit $E$ et $F$.

(20) Le titre de flamen civitatis est exceptionnel en Narbonnaise, mais on le rencontre souvent ailleurs; en Gaule on peut citer I.yon, C. I. L. XIII, 1674, 1675, flamen II vir in civilale Sequanorum.

(21) C. I. L. XIII, 2211, 2212, 2213, 4001, 6103, 6366, 7090.

(22) C. I. L. XII, 599 à Julhans ; 829 à Arles ; 1317 à Vaison ; I363 à Saint-Maurice de Dròme ; 1985 à Vienne ; 2737 près de Bagnols; 2837 à Ugernum ; 2919 à Brignon ; 3256, 3257, 3429, 3746, 3747, 3748, 3749 à Nimes ; 4363, 4960,4989 à Narbonne.

(23) C. I. L. XII, 829.

(24) Le nom de Serenus apparaît à Aix, C. I. L. XII, 499; à Vaison, 1452 ; à Die 1556 ; à Narbonne, 4422. On ne Ie rencontre dans les Trois Gaules que comme cognomen.

(25) A Londres, C. I. I. VII, 1331

(26) C. I. L. XII, 2339, 2415, 2935, 3471, 3472, 1870, 4484. Le C. I. L. XIII ne signale pas ce nom dans les trois Gaules.

(27) C. I. L. XIII, 1791 II 21 et 6980. 
Marcus Marinius ${ }^{28}$ Mateclanus, celui qui a rédigé le décret ${ }^{29}$, porte un cognomen vraisemblablement celtique, à rapprocher de Mateiclus déjà connu ${ }^{30}$.

Avec la septième ligne commencent les lacunes qui privent le texte de ses parties essentielles, où étaient indiqués les motifs et les résolutions du décret.

[1. 7-8] Cum M(arcus) Val(erius) Licinianus, M(arcus)... [v]iri retulissent aed[ilium] et quaest $[$ oris $]$ prae [clarum $] f(a c i n u s] \ldots . . . \ldots[$ posteris $]$ (p)erhiberi debere.

Dans ces deux lignes, le graveur a commis deux erreurs de transcription, relulissent pour retulissent et $e i$ pour $e l$; elles contenaient l'exposé fait par deux autres magistrats, les duoviri du municipe, de la conduite mémorable des édiles et du ou des questeurs; belle action dont le souvenir devait être rappelé à la postérité.

En reconnaissance, l'assemblée des décurions décréta en leur faveur des honneurs ou privilèges dont on ne peut plus connaître la nature, les lacunes étant trop étendues pour qu'on risque une restitution :

[1. 9-15] Placuit ${ }^{31} . . . \ldots$. ipsorum el quos inc....... T(itus) Veranius L... niam quia.......... Veranius........ am iusti.... um Lic....

Il est vraisemblable que $T$. Veranius $L \ldots$, deux fois cité, était l'un des magistrats bénéficiaires du décret; toute autre hypothèse serait par trop hasardeuse.

[1. 16-18] Sext(us) Sa....... Salu........ qua.......

Sur ces trois dernières lignes devaient figurer les noms des témoins.

Malgré l'état fragmentaire dans lequel il nous est parvenu, le décret des décurions de Digne reste un document exceptionnel ; il éclaire la condition juridique d'une ville sur laquclle les sources littéraires demeurent à peu près muettes. Son organisation municipale se dessine, avec sa curie, ses édiles, son ou ses questeurs et ses duoviri; assemblée où siège le Flamine dont l'action spirituelle s'étend à toute la cité. Son nom officiel se complète et bien qu'à ce point de vue les éléments nouveaux soient encore énigmatiques, ils sont peut-être un gage de loyalisme ayant mérité l'attention de l'Empereur ${ }^{32}$.

\section{Henri Rolland.}

(28) Patronyme cité au C. I. L. XIII, 5291, 2015, 7564, 2538, 8231.

(29) Faut-il voir dans scripsit la "préparation matérielle " de la gravure sur bronze et non l'" élaboration intellectuelle "(MaLLon, o. c., p. 57) ? je pense que, dans le cas présent, scripsil a prècédé l'ordinatio du modèle destiné au graveur.

(30) C. I. I. X, 6505.

(31) Peut-être après placuil peut-on compléter par universis, comme sur le décret de Cumes, C. I. L. X, 3698 : ...placuit universis. Comparer aussi C. I. L. XIV, 2795, placuit in tabula aera scribi.

(32) Qu'il nous soit permis d'exprimer notre vive gratitude à M. le Professeur André Piganiol, qui a bien voulu s'intéresser à cette découverte et nous faire profiter de ses savantes suggestions, ainsi qu'à MM. Delaye, R. Collier et R. Moulin, qui ont bien voulu me laisser le soin de la publier. 